\title{
Diversidad y distribución de acridios (Orthoptera: Acridoidea) en pastizales del sur de la región pampeana, Argentina
}

\author{
Yanina Mariottini ${ }^{1}$, María Laura De Wysiecki ${ }^{1} \&$ Carlos Ernesto Lange ${ }^{1,2}$ \\ 1. Centro de Estudios Parasitológicos y de Vectores (CEPAVE) (CCT La Plata-CONICET-UNLP), Calle 2 №584, CP \\ 1900, La Plata, Argentina; ymariottini@cepave.edu.ar,mlw@cepave.edu.ar, carlos1@cepave.edu.ar \\ 2. Comisión de Investigaciones Científicas (CIC) de la provincia de Buenos Aires.
}

Recibido 29-XI-2011. Corregido 20-IX-2012. Aceptado 05-X-2012.

\begin{abstract}
Diversity and distribution of grasshoppers (Orthoptera: Acridoidea) in grasslands of the Southern Pampas region, Argentina. In Argentina, the grasslands of Pampas region comprise approximately $15 \%$ of the country. As in other grasslands of the world, grasshoppers are among the most important native herbivores. Their economic importance has been recognized in Argentina since the mid to late nineteenth century, since outbreaks of different species have become recurrent phenomena. Therefore, the main objective of this work was to study their diversity and distribution in grasslands of the Southern Pampas region (Laprida county, Buenos Aires province), as one of the most affected areas. The study was conducted during five seasons (2005-10). Sampling sites were represented by the most common plant communities in this area, classified in four categories: native grasslands, disturbed grasslands, implanted pastures and halophilous grasslands. The samplings were conducted from mid-spring to early autumn, with five or six samples per season. We estimated the following population descriptors: species richness (S), eveness (E), dominance (J), and diversity index $\left(\mathrm{H}^{\prime}\right)$. In order to evaluate the similitude of the grasshopper communities present in the different plant communities, we used qualitative and quantitative coefficients of similitude. A total of 22 species of grasshoppers were collected, of which 21 belong to the family Acrididae. The subfamily Melanoplinae was the most diverse with eight species. The largest species richness was recorded in native grasslands (18). The different communities of grasshoppers had similar indices of evenness and dominance $(\mathrm{p}>0.05)$. Considering all plant communities, the average value of Shannon-Wiener index was $1.58 \pm 0.075$. There was a positive correlation between evenness index and species richness $(\mathrm{p}<0.05)$. The diversity index $\mathrm{H}^{\prime}$ was different between plants communities $(\mathrm{p}<0.05)$, and it was higher in the disturbed grassland $(1.75 \pm 0.096, \mathrm{p}<0.05)$ than in the halophilous grasslands $(1.34 \pm 0.12)$. Native and disturbed grasslands had a higher plant richness than halophilous grasslands and implanted pastures $(\mathrm{p}<0.05)$. There was a positive relationship between plant richness and grasshoppers species richness, and diversity of grasshoppers. According to the qualitative indices applied, the similitude between different grasshopper communities was higher than $60 \%$. In general, the species that had a higher frequency of occurrence showed greater abundance and distribution. Covasacris pallidinota, Dichroplus elongatus, D. maculipennis, Borellia bruneri and B. pallida were the most widely distributed species, most of them (12) showed a restricted distribution and few (five) an intermediate distribution. Rev. Biol. Trop. 61 (1): 111-124. Epub 2013 March 01.
\end{abstract}

Key words: grasshoppers, grasslands, species richness, diversity index, distribution.

En los ecosistemas de pastizal, los acridios constituyen uno de los grupos de insectos herbívoros más importantes, cumpliendo un rol fundamental como consumidores primarios y componentes de las cadenas tróficas en el ciclado de los nutrientes y de la energía (Belovsky 2000, Branson et al. 2006). Asimismo, algunas especies son plagas y en años de explosiones poblacionales (outbreaks) compiten con el ganado y la fauna silvestre por el forraje y destruyen diversos cultivos, causando grandes pérdidas económicas a los productores (Fielding \& Brusven 1995, Branson et al. 2006, Carbonell et al. 2006, Mariottini et al. 2011a). 
La diversidad de los acridios está determinada por una compleja relación entre factores extrínsecos como el clima y la estructura y composición específica de la vegetación (Sánchez \& De Wysiecki 1993, Kemp \& Cigliano 1994, Cigliano et al. 2000) y factores intrínsecos, como la dinámica poblacional de las diferentes especies (Joern 2005, Zhong-Wei et al. 2006). Las comunidades de acridios suelen exhibir una gran variabilidad en la composición y abundancia de especies (Joern \& Gaines 1990, Jonas \& Joern 2007, De Wysiecki et al. 2011). Ronderos (1985) señala que la coexistencia de diferentes especies de acridios en pastizales se debe a la interacción de varios factores como: estrategias reproductivas, diferencias interespecíficas en la diapausa, selección del alimento, fragmentación del hábitat y desarrollo secuencial de especies competidoras.

Uno de los aspectos más importantes a considerar cuando se aborda el estudio de la diversidad en los pastizales, es el impacto que las actividades antrópicas generan sobre la diversidad de estos ecosistemas. La ganadería y la agricultura producen profundos cambios en los ecosistemas de pastizal, modificando el funcionamiento y la diversidad estructural y florística de dichos sistemas (Sala et al. 1986, Milchunas \& Lauenroth 1993, Nai-Bregaglio et al. 2002). En general, como consecuencia de estas actividades, la diversidad de las comunidades vegetales disminuye, generando una simplificación de especies en las comunidades de acridios asociadas (Zhong-Wei et al. 2006). Esta situación se observa frecuentemente en pastizales naturales sobrepastoreados (Joern 2005, Pfadt 1982) y en cultivos donde la riqueza de especies vegetales disminuye al mínimo y la diversidad de insectos suele ser menor (Root 1973, Altieri 1983, Sánchez \& De Wysiecki 1993).

Los pastizales de la región pampeana representan aproximadamente el $15 \%$ de la superficie de la Argentina. Durante el siglo pasado esta región fue el escenario del desarrollo de la industria ganadera así como de la agricultura (Soriano et al. 1992). Muchos de los pastizales originales fueron reemplazados por cultivos y pasturas implantadas. Asimismo, los múltiples efectos del sobrepastoreo bovino determinaron cambios en la composición florística, donde los pastos nativos son desplazados por dicotiledóneas exóticas de bajo valor forrajero (Sala et al. 1986, Facelli 1988). En las últimas décadas en la mayoría de estos ambientes se ha incrementado el uso del suelo, las actividades agrícola-ganaderas se han intensificado y las áreas de pastizales naturales han sido drásticamente reducidas o alteradas (Chaneton 2005, Ghersa 2005, Omancini et al. 2005).

El partido de Laprida se encuentra en la parte más austral de la sub-región de la Pampa Deprimida y comprende una llanura que se extiende a hasta $210 \mathrm{msnm}$, entre las Sierras de Tandilla al NE y las Sierras de Ventania al Sur (Batista et al. 1988). Los pastizales constituyen la vegetación predominante de la región pampeana (Chaneton 2005, Insausti et al. 2005), pero debido a limitaciones de los suelos, la actividad dominante es la ganadera (Perelman et al. 2001, Batista et al. 2005). Desde fines del siglo XIX los pastizales de la sub-región de la pampa deprimida están sometidos a un intenso pastoreo por ganado vacuno, principalmente, lo que mantiene su fisonomía relativamente homogénea a través del paisaje (Batista et al. 2005).

Considerando la importancia de los acridios como componentes nativos de los pastizales, y que dichos sistemas han sido modificados debido a la intensificación de las actividades agrícola-ganaderas, el objetivo de este trabajo fue estudiar la diversidad y la distribución de los acridios en las comunidades vegetales más características del Sur de la región pampeana, área afectada históricamente por estos insectos.

\section{MATERIALES Y MÉTODOS}

Área de estudio: El área de estudio estuvo localizada en el partido de Laprida ubicado en el Sur de la región pampeana (sub-región de la Pampa Deprimida), provincia de Buenos Aires $\left(36^{\circ} 02^{\prime} \mathrm{S}-59^{\circ} 06^{\prime} \mathrm{W}\right)$. La temperatura media anual es de $14^{\circ} \mathrm{C}$, La temperatura media en verano es de $22^{\circ} \mathrm{C}$ y en invierno de $6^{\circ} \mathrm{C}$. La 
precipitación media anual oscila entre $800 \mathrm{y}$ $900 \mathrm{~mm}$ configurando un régimen hídrico subhúmedo (Sala et al. 1981).

Sitios de muestreo: Los sitios de muestreo se seleccionaron teniendo en cuenta las comunidades vegetales características de la zona. Los mismos se clasificaron en cuatro categorías de acuerdo a la vegetación dominante, la cual refleja la historia de uso del área (Fielding \& Brusven 1993, Torrusio et al. 2002): pastizales nativos (dominados por gramíneas), pastizales halófilos (dominados por especies de pastos nativas que crecen en suelos salinos, como por ejemplo Distichlis spicata), pasturas implantadas (dominadas por especies del género Agropyron) y pastizales alterados (pastizales nativos sometidos a pastoreo por ganado). Para cada categoría se seleccionaron tres sitios dando un total de 12 sitios (Cuadro 1), que se mantuvieron constantes durante todo el estudio. En cada sitio se estimó la cobertura de las diferentes especies vegetales, siguiendo el método de Daubenmire (1959) de amplia utilización en el estudio de comunidades herbáceas. Las plantas que no pudieron ser identificadas en el campo

\section{CUADRO 1}

Ubicación geográfica de los 12 sitios de estudio, en el partido de Laprida, Buenos Aires

TABLE 1

Geographical location of the 12 study sites in Laprida County, Buenos Aires

\begin{tabular}{|c|c|c|}
\hline $\begin{array}{c}\text { Comunidad } \\
\text { vegetal }\end{array}$ & Sitios & Coordenadas \\
\hline \multirow{3}{*}{ P. Halófilos } & 1 & $37^{\circ} 45^{\prime} 11.48^{\prime \prime} \mathrm{S}-60^{\circ} 44^{\prime} 34.1^{\prime \prime} \mathrm{W}$ \\
\hline & 7 & $37^{\circ} 25^{\prime} 42.6^{\prime \prime} \mathrm{S}-60^{\circ} 55^{\prime} 10.4^{\prime \prime} \mathrm{W}$ \\
\hline & 11 & $37^{\circ} 44^{\prime} 33.2^{\prime \prime} \mathrm{S}-60^{\circ} 43^{\prime} 52.1^{\prime \prime} \mathrm{W}$ \\
\hline \multirow[t]{3}{*}{ P. Implantadas } & 2 & $37^{\circ} 44^{\prime} 31.4^{\prime \prime} \mathrm{S}-60^{\circ} 44^{\prime} 13.3^{\prime \prime} \mathrm{W}$ \\
\hline & 6 & $37^{\circ} 25^{\prime} 42.6^{\prime \prime} \mathrm{S}-60^{\circ} 55^{\prime} 36.2^{\prime \prime} \mathrm{W}$ \\
\hline & 10 & $37^{\circ} 44^{\prime} 21.6^{\prime \prime} \mathrm{S}-60^{\circ} 43^{\prime} 46.4^{\prime \prime} \mathrm{W}$ \\
\hline \multirow[t]{3}{*}{ P. Alterados } & 3 & $37^{\circ} 44^{\prime} 15^{\prime \prime} \mathrm{S}-60^{\circ} 43^{\prime} 53.1^{\prime \prime} \mathrm{W}$ \\
\hline & 8 & $37^{\circ} 44^{\prime} 52.82^{\prime \prime} \mathrm{S}-60^{\circ} 44^{\prime} 7.48^{\prime \prime} \mathrm{W}$ \\
\hline & 9 & $37^{\circ} 25^{\prime} 16.1^{\prime \prime} \mathrm{S}-60^{\circ} 55^{\prime} 02.2^{\prime \prime} \mathrm{W}$ \\
\hline \multirow[t]{3}{*}{ P. Nativos } & 4 & $37^{\circ} 45^{\prime} 09.8^{\prime \prime} \mathrm{S}-60^{\circ} 44^{\prime} 08.5^{\prime \prime} \mathrm{W}$ \\
\hline & 5 & $37^{\circ} 34^{\prime} 19.7^{\prime \prime} \mathrm{S}-60^{\circ} 45^{\prime} 51^{\prime \prime} \mathrm{W}$ \\
\hline & 12 & $37^{\circ} 44^{\prime} 55.9^{\prime \prime} \mathrm{S}-60^{\circ} 44^{\prime} 02.4^{\prime \prime} \mathrm{W}$ \\
\hline
\end{tabular}

fueron herborizadas y trasladadas al laboratorio para su identificación. Los muestreos de la vegetación se realizaron en noviembre y diciembre 2005 y enero 2006, coincidiendo con el momento de mayor productividad vegetal.

Muestreo de acridios: Los muestreos se realizaron desde mediados de primavera hasta principios de otoño, se efectuaron entre cinco y seis muestreos por temporada. En 2005-06 los muestreos se realizaron desde noviembre hasta marzo, en 2006-07 los muestreos fueron en octubre, diciembre, enero, febrero, marzo y abril. En 2007-08 se realizaron desde octubre hasta marzo, en 2008-09 se muestreó en noviembre, diciembre, enero, febrero y abril, y en la última temporada 2009-10 los muestreos fueron en octubre, noviembre, enero, febrero y abril. Se tomaron un total de 321 muestras. La composición específica se determinó mediante 200 golpes o barridos con redes entomológicas de $40 \mathrm{~cm}$ de diámetro y $75 \mathrm{~cm}$ de profundidad, a lo largo de cuatro transectas de aproximadamente $3 \mathrm{~m}$ de ancho y $50 \mathrm{~m}$ de longitud cada una, en cada uno de los 12 sitios. Cada golpe consistió en un arco de $180^{\circ}$ a través de la vegetación, método de eficacia probada para la obtención de muestras representativas de la comunidad (Larson et al. 1999). Los acridios colectados se colocaron en bolsas plásticas y se conservaron en heladeras a $4^{\circ} \mathrm{C}$, hasta ser llevados al laboratorio para su identificación (especie y estado de desarrollo). Para los diferentes sitios, en cada momento de muestreo, se estimó la riqueza de especies (S), el índice de equitatividad de Pielou (E), el índice de dominancia de Simpson (J), y el índice de diversidad de Shannon-Wiener $\left(\mathrm{H}^{\prime}\right)$. A fin de evaluar la similitud de las comunidades de acridios presentes en las diferentes comunidades vegetales, se utilizaron coeficientes de similitud cualitativos (Jaccard y Sorensen) y cuantitativo de Sorensen.

La distribución de las especies de acridios se determinó a partir de la proporción de sitios en los que cada especie fue registrada, en relación al total de sitios muestreados a lo largo de todo el período de estudio. Se generó una escala de distribución con cuatro categorías: 
a- (1-25\%: distribución restringida), b- (26$50 \%$ : distribución intermedia), c- (51-75\%: distribución amplia) y d- (>75\%: distribución muy amplia).

A fin de corroborar las categorías de vegetación dominante propuestas inicialmente se realizó un análisis de ordenación "Detrended Correspondence Analysis" (DCA) (Hill \& Gauche 1980). Para dicho análisis se consideraron diferentes características de las especies vegetales como por ejemplo, si son plantas perennes o anuales, nativas o introducidas. Las variables de la vegetación utilizadas fueron los porcentajes de cobertura de: gramíneas nativas perennes, especies halófilas, especies dicotiledóneas nativas perennes y anuales, dicotiledóneas introducidas anuales y perennes y el porcentaje de pasturas perennes (Torrusio et al. 2002).

La riqueza de especies de plantas entre las diferentes comunidades vegetales se comparó mediante un test de Kruskal-Wallis, seguido del test U de Mann-Whitney.

La diversidad $\left(\mathrm{H}^{\prime}\right)$, la equitatividad (E) y el índice de dominancia (J) de especies de acridios entre las diferentes comunidades vegetales se compararon mediante un análisis de la varianza (ANOVA) de una vía. Previamente, se utilizó la prueba de Levenne para corroborar la homogeneidad de las varianzas y a posteriori se empleó la prueba de Tukey para la comparación de medias.

La relación entre el índice de equidad y la riqueza de especies de acridios de los diferentes sitios se estimó mediante un análisis de correlación.

Se estimó la relación entre la riqueza de especies de plantas y la riqueza de acridios y el índice $\mathrm{H}^{\prime}$ de Shannon-Wiener mediante un análisis de regresión simple.

A fin de examinar la relación entre el logaritmo natural de la abundancia relativa promedio y el número de sitios-año en los que cada especie fue colectada, se realizó un análisis de regresión simple para todos los sitios teniendo en cuenta las cinco temporadas de estudio (Kemp, 1992a). Todos los análisis estadísticos se realizaron con el programa XLSTAT 7.5.3 (Addinsoft 2005).

\section{RESULTADOS}

El DCA confirmó las diferentes categorías determinadas para la vegetación de acuerdo a las características de las plantas dominantes. Los dos primeros ejes explicaron el $64.5 \%$ de la variación (Fig. 1). En los pastizales nativos, el $80.2 \%$ de la cobertura vegetal fueron especies nativas perennes, representado las gramíneas el 63.4\% (Stipa formicarum, Panicum bergii, Stipa trichotoma, Stipa caudata, Festuca pampeana, Mellica eremophyla, Eleusine tristachya, entre otras) y las dicotiledóneas nativas perennes el 19.8\% (Eryngium nudicaule, Phyla canescens, Plantago berroi, entre otras) (Cuadro 2). Los pastizales halófilos estuvieron ampliamente dominados por Distichlis spicata (78\%). En las pasturas, la cobertura vegetal estuvo mayormente representada por Agropyron elongatum, encontrándose también un porcentaje importante de especies halófilas, como acompañantes. Los pastizales alterados tuvieron el mayor porcentaje de dicotiledóneas exóticas $(50.15 \%)$, de las cuales el $47.4 \%$ correspondió a dicotiledóneas introducidas anuales (Cardus pycnocephalus, Centaurea calcitrapa, Ammi majus, Cardus acanthoides) (Cuadro 2).

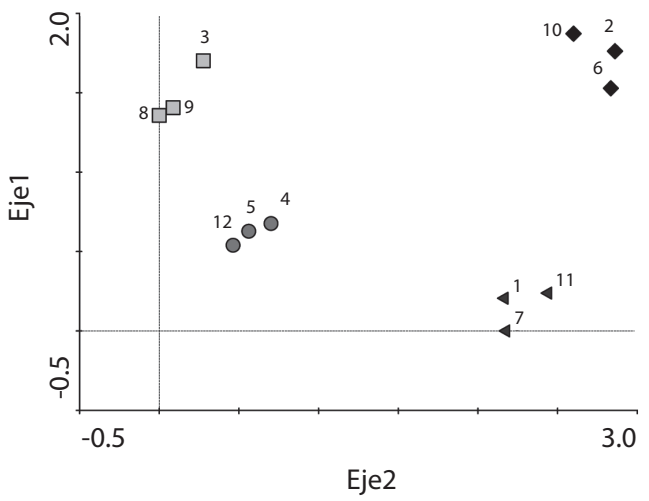

Fig. 1. Resultado del DCA aplicado a los sitios de estudio. Pastizales halófilos (Sitios: 1,7, 11), pastizales nativos (Sitios: 4, 5 y 12), pasturas implantadas (Sitios: 2, 6 y 10), y pastizales alterados (Sitios: 3,8 y 9 ).

Fig. 1. Results of DCA applied to the study sites. Halophilous grasslands (Sites: 1, 7 and 11), native grasslands (Sites: 4, 5 and 12), planted pastures (Sites: 2, 6 and 10), and disturbed grasslands (Sites: 3, 8 and 9). 
CUADRO 2

Valores promedios y rangos de las variables de vegetación por comunidad vegetal en Laprida, Buenos Aires

TABLE 2

Mean values and ranges of vegetation variables in plant communities in Laprida, Buenos Aires

\begin{tabular}{lcccc}
\multicolumn{1}{c}{ Variables de vegetación } & P. Halófilos & P. Implantadas & P. Nativos & P. Alterados \\
Gramíneas perennes nativas & $9.05(0-80)$ & $0.6(0-7.14)$ & $63.4(20-97)$ & $19.72(0-76)$ \\
Pasturas perennes & $7.5(0-80)$ & $76(20-100)$ & $4.86(0-14.3)$ & $2.10(0-14.28)$ \\
Especies halófilas & $78(20-95)$ & $17.8(0-40)$ & 0 & $1.15(0-7.14)$ \\
Dicot. anuales introducidas & $1.19(0-14.3)$ & $5(0-40)$ & $2.19(0-15)$ & $47.4(1.33-80)$ \\
Dict. perennes introducidas & 0 & 0 & $3.94(0-18.2)$ & $3.75(0-16.66)$ \\
Dicot. anuales nativas & $3.16(0-16)$ & $2.5(0-30)$ & $1.25(0-15)$ & $5.27(0-40)$ \\
Dict. perennes nativas & 0 & 0 & $19.8(0-50)$ & $9.95(0-50)$ \\
\hline
\end{tabular}

La riqueza de especies de las comunidades vegetales fue significativamente diferente (Kruskal-Wallis: Ho=17.1, p=0.001, g.l=3), siendo los pastizales nativos y los pastizales alterados los que presentaron mayor riqueza (U Mann Whitney $\mathrm{p}<0.05$ ) (Cuadro 3).

La riqueza acumulada de acridios fue de 22 especies pertenecientes a la superfamilia Acridoidea, 21 de ellas correspondientes a la familia Acrididae y una a Romaleidae (Cuadro 4). En los distintos pastizales, la subfamilia Melanoplinae fue la más diversa, se recolectaron ocho especies a lo largo del estudio. En promedio, se recolectaron por temporada en los pastizales halófilos 5.4 especies de melanoplinos; error estándar 0.4; rango (7-5),

\section{CUADRO 3 \\ Riqueza de plantas promedio ( $\pm \mathrm{ES}$ ) y rango en las diferentes comunidades vegetales de Laprida, Buenos Aires, en el período 2005-2010}

TABLE 3

Average plant richness $( \pm \mathrm{SE})$ in different plant communities of Laprida, Buenos Aires, during 2005-2010

\begin{tabular}{lcc} 
& S de plantas promedio & $\begin{array}{c}\text { U de Mann } \\
\text { Whitney }\end{array}$ \\
P. Halófilos & $5.3 \pm 0.30(3-9)$ & $\mathrm{b}$ \\
P. Implantadas & $4 \pm 0.63(2-6)$ & $\mathrm{b}$ \\
P. Alterados & $12 \pm 0.21(9-15)$ & $\mathrm{a}$ \\
P. Nativos & $12.3 \pm 0.75(10-15)$ & $\mathrm{a}$ \\
\hline
\end{tabular}

* Letras distintas indican diferencias significativas (U de Mann Whitney $\mathrm{p}<0.05$ ) en las pasturas implantadas $5.4 ; 0.24 ;(6-5)$, en los pastizales alterados $6 ; 0.32 ;(7-5)$ y en los pastizales nativos 6 ; 0.55 ; (7-4). La segunda Subfamilia más diversa fue Gomphocerinae, colectándose siete especies. El promedio de especies de gonfocerinos colectados por temporada en los pastizales halófilos fue 2.6; 0.4; (4-2), en las pasturas implantadas 3.2; 0.2; (4-3), en los pastizales alterados y en los pastizales nativos 3.6 ; $0.51 ;(5-2)$.

El mayor número de especies de acridios se registró en los pastizales nativos, con un valor máximo de 18 en la temporada 2005-06 y un mínimo de 10 en la temporada 2009-10 (Cuadro 5). Los pastizales halófilos fueron las comunidades con el menor número de especies, con un máximo de 12 en las temporadas 2005-06 y 2006-07 y de nueve en la temporada 2009-10 (Cuadro 5). En promedio, en los pastizales nativos se recolectaron 14; 1.30 especies/ temporada, en los pastizales halófilos 10.8; 0.58 , en las pasturas implantadas: $12 ; 0.63$ y en los pastizales alterados $12.8 ; 1.32$.

Los valores del índice de equidad de acridios entre las diferentes comunidades vegetales no fueron significativamente diferentes (Levene: $\mathrm{F}=0.36, \mathrm{gl}=3.56, \mathrm{p}=0.78$; ANOVA: $\mathrm{F}=1.84$, $\mathrm{gl}=3.56, \mathrm{p}=0.15)$. El valor promedio en los pastizales halófilos fue de 0.56 ; 0.05 ; $(0.65$ 0.41 ), en las pasturas implantadas de 0.62 ; $0.031(0.73-0.64)$, en los pastizales alterados de $0.69 ; 0.028(0.77-0.60)$, y en los pastizales nativos de $0.63 ; 0.062(0.74-0.39)$. 
CUADRO 4

Especies de acridios recolectadas, distribución y abundancia relativa promedio de las mismas, durante 2005-2010 en Laprida, Buenos Aires

TABLE 4

Grasshopper species collected their distribution and mean relative abundance during 2005-2010 in Laprida, Buenos Aires

\begin{tabular}{|c|c|c|c|c|}
\hline Especies de acridios & $\mathrm{F}$ & $\begin{array}{c}\% \text { sitios con registro } \\
\text { de la especie }\end{array}$ & $\begin{array}{l}\text { Abundancia relativa }(\%) \\
\text { promedio } \pm \mathrm{ES}\end{array}$ & $\begin{array}{l}\text { Escala de } \\
\text { distribución }\end{array}$ \\
\hline \multicolumn{5}{|l|}{ Acrididae } \\
\hline \multicolumn{5}{|l|}{ Acridinae } \\
\hline Covasacris pallidinota (Bruner) & 208 & 64.8 & $18.4 \pm 1.33$ & $\mathrm{c}$ \\
\hline Parorphula graminea Bruner & 36 & 11.2 & $0.3 \pm 0.06$ & a \\
\hline Cocytotettix argentina (Bruner) & 25 & 7.8 & $1.65 \pm 0.64$ & a \\
\hline \multicolumn{5}{|l|}{ Copiocerinae } \\
\hline Aleuas lineatus Stål & 97 & 30.2 & $3.3 \pm 0.44$ & $\mathrm{~b}$ \\
\hline Aleuas vitticollis Stål & 11 & 3.4 & $0.33 \pm 0.11$ & a \\
\hline \multicolumn{5}{|l|}{ Leptysminae } \\
\hline Tucayaca gracilis (Giglio-Tos) & 3 & 0.9 & $0.06 \pm 0.032$ & a \\
\hline \multicolumn{5}{|l|}{ Melanoplinae } \\
\hline Baeacris punctulatus (Thumberg) & 2 & 0.6 & $0.03 \pm 0.02$ & a \\
\hline Baeacris pseudopunctulatus (Ronderos) & 85 & 26.5 & $3.23 \pm 0.7$ & $\mathrm{~b}$ \\
\hline Dichroplus conspersus Bruner & 113 & 35.2 & $2.71 \pm 0.31$ & $\mathrm{~b}$ \\
\hline Dichroplus elongatus Giglio-Tos & 213 & 66.4 & $12.7 \pm 1.4$ & $\mathrm{c}$ \\
\hline Dichroplus maculipennis (Blanchard) & 171 & 53.3 & $15.7 \pm 1.24$ & $\mathrm{c}$ \\
\hline Dichroplus pratensis Bruner & 89 & 27.7 & $2.45 \pm 0.37$ & $\mathrm{~b}$ \\
\hline Leiotettix pulcher Rehn & 2 & 0.6 & $0.034 \pm 0.03$ & a \\
\hline Scotussa lemniscata (Stål) & 62 & 19.3 & $2.7 \pm 0.47$ & a \\
\hline \multicolumn{5}{|l|}{ Gomphocerinae } \\
\hline Borellia bruneri (Rehn) & 232 & 72.3 & $24 \pm 1.24$ & $\mathrm{c}$ \\
\hline Borellia pallida (Bruner) & 172 & 53.6 & $3.52 \pm 0.28$ & $\mathrm{c}$ \\
\hline Scyllinula variabilis (Bruner) & 1 & 0.3 & $0.015 \pm 0.015$ & a \\
\hline Sinipta dalmani (Stål) & 85 & 26.5 & $8.14 \pm 1.3$ & $\mathrm{~b}$ \\
\hline Staurorhectus longicornis Giglio-Tos & 14 & 4.4 & $0.63 \pm 0.25$ & a \\
\hline Amblytropidia australis Bruner & 1 & 0.3 & $0.003 \pm 0.003$ & $\mathrm{a}$ \\
\hline Orphulella punctata (De Geer) & 1 & 0.3 & $0.007 \pm 0.007$ & a \\
\hline \multicolumn{5}{|l|}{ Romaleidae } \\
\hline \multicolumn{5}{|l|}{ Romaleinae } \\
\hline Diponthus argentinus Pictet and Saussure & 2 & 0.6 & $0.011 \pm 0.008$ & $\mathrm{a}$ \\
\hline
\end{tabular}

F: cantidad de sitios en los que se registró la especie. Escala de distribución: a: restringida, b: intermedia, c: amplia, d: muy amplia.

Existió una correlación significativa entre la cantidad de especies de acridios de las diferentes comunidades vegetales (S) y el índice de equidad (E) $(\mathrm{F}=13.95$, g.l 1.58 , $\mathrm{p}=0.0004$ ) (Fig. 2).

El índice de dominancia promedio en los pastizales halófilos fue de $0.35 ; 0.054$, en las pasturas implantadas de $0.31 ; 0.037$, en los pastizales alterados de $0.25 ; 0.031 \mathrm{y}$ en los pastizales nativos de $0.29 ; 0.072$. No se registraron diferencias significativas entre los valores del índice de dominancia para acridios en las diferentes comunidades vegetales (Levene: $\mathrm{F}=0.136, \mathrm{gl}=3.56, \mathrm{p}=0.94$; ANOVA: $\mathrm{F}=1.15$, $\mathrm{gl}=3.56, \mathrm{p}=0.337$ ).

Considerando todos los ambientes, el valor promedio del índice $\mathrm{H}^{\prime}$ Shannon-Wiener fue de $1.58 ; 0.075$. La diversidad de acridios entre las 
CUADRO 5

Índices de diversidad estimados para las comunidades de acridios presentes en las diferentes comunidades vegetales de Laprida, Buenos Aires, por temporada (2005-2010)

TABLE 5

Diversity indices estimated for grasshopper communities in different plant communities in Laprida, Buenos Aires, by season (2005-2010)

\begin{tabular}{|c|c|c|c|c|c|}
\hline Temporadas & Índices & P. Halófilos & P. Implantadas & P. Alterados & P. Nativos \\
\hline \multirow[t]{5}{*}{ 2005-06 } & $\mathrm{N}$ & 943 & 628 & 810 & 884 \\
\hline & $\mathrm{S}$ & 12 & 13 & 17 & 18 \\
\hline & $\mathrm{E}$ & 0.5 & 0.73 & 0.68 & 0.72 \\
\hline & $\mathrm{J}$ & 0.41 & 0.19 & 0.22 & 0.18 \\
\hline & $\mathrm{H}^{`}$ & 1.24 & 1.88 & 1.95 & 2.06 \\
\hline \multirow[t]{5}{*}{ 2006-07 } & $\mathrm{N}$ & 2507 & 959 & 645 & 1078 \\
\hline & $\mathrm{S}$ & 12 & 13 & 13 & 16 \\
\hline & $\mathrm{E}$ & 0.60 & 0.63 & 0.73 & 0.74 \\
\hline & $\mathrm{J}$ & 0.26 & 0.29 & 0.21 & 0.17 \\
\hline & $\mathrm{H}^{\top}$ & 1.50 & 1.62 & 1.87 & 2.06 \\
\hline \multirow[t]{5}{*}{ 2007-08 } & $\mathrm{N}$ & 2471 & 1615 & 1780 & 1910 \\
\hline & $\mathrm{S}$ & 10 & 13 & 14 & 15 \\
\hline & $\mathrm{E}$ & 0.65 & 0.60 & 0.67 & 0.66 \\
\hline & $\mathrm{J}$ & 0.27 & 0.31 & 0.23 & 0.26 \\
\hline & $\mathrm{H}^{\top}$ & 1.51 & 1.55 & 1.78 & 1.80 \\
\hline \multirow[t]{5}{*}{ 2008-09 } & $\mathrm{N}$ & 2297 & 1473 & 1618 & 1487 \\
\hline & $\mathrm{S}$ & 11 & 11 & 10 & 12 \\
\hline & $\mathrm{E}$ & 0.65 & 0.61 & 0.77 & 0.65 \\
\hline & $\mathrm{J}$ & 0.26 & 0.36 & 0.20 & 0.29 \\
\hline & $\mathrm{H}^{\top}$ & 1.56 & 1.47 & 1.77 & 1.61 \\
\hline \multirow[t]{5}{*}{ 2009-10 } & $\mathrm{N}$ & 1891 & 1569 & 1516 & 2050 \\
\hline & $\mathrm{S}$ & 9 & 10 & 10 & 10 \\
\hline & $\mathrm{E}$ & 0.41 & 0.54 & 0.60 & 0.39 \\
\hline & $\mathrm{J}$ & 0.53 & 0.41 & 0.37 & 0.57 \\
\hline & $\mathrm{H}^{\prime}$ & 0.89 & 1.24 & 1.39 & 0.91 \\
\hline
\end{tabular}

$\mathrm{N}$ : número de individuos, S: riqueza de especies, E: índice de Equitatividad, J: índice de Dominancia, $\mathrm{H}^{\prime}$ : índice de Diversidad de Shannon-Wiener.

distintas comunidades vegetales fue significativamente diferente (Levene: $\mathrm{F}=0.19, \mathrm{gl}=3.56$, $\mathrm{p}=0.99$; ANOVA: $\mathrm{F}=3.32, \mathrm{gl}=3.56, \mathrm{p}=0.026$ ), siendo la diversidad de los pastizales alterados significativamente superior a la de los pastizales halófilos (Tukey p=0.025) (Fig. 3).

Existió una relación significativa entre la riqueza de plantas promedio y la riqueza de acridios $\left(\mathrm{R}^{2}=0.36, \mathrm{~F}=5.73, \mathrm{p}=0.038\right)$ (Fig. 4), y el índice de diversidad $\mathrm{H}^{\prime}$ de Shannon-Wiener $\left(\mathrm{R}^{2}=0.67, \mathrm{~F}=20.53, \mathrm{p}=0.001\right)$ (Fig. 5), siendo mayores los valores en los sitios que presentaron una mayor riqueza de especies de plantas.

Las similitudes cualitativas, estimadas por los índices de Jaccard y Sørensen, entre las comunidades de acridios de las diferentes comunidades vegetales fueron altas, superiores al 60\% (Cuadro 6). Los pastizales halófilos y las pasturas implantadas tuvieron las comunidades de acridios más similares. El índice cuantitativo de Sørensen, que además de la presencia de las especies tiene en cuenta su 


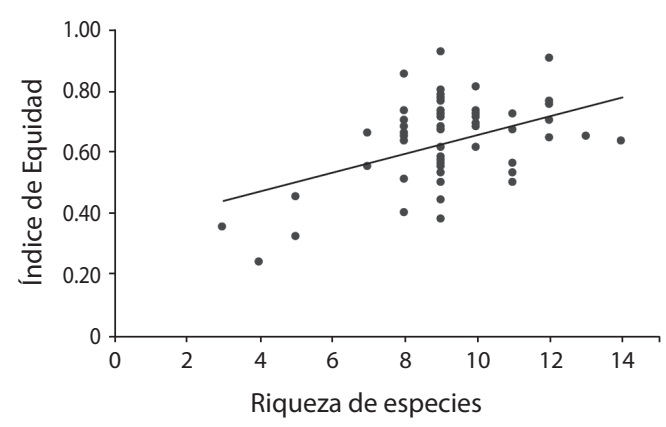

Fig. 2. Correlación entre el índice de equidad y la riqueza de especies de acridios en Laprida, Buenos Aires, 20052010 .

Fig. 2. Correlation between eveness index and species richness of grasshoppers in Laprida, Buenos Aires, 20052010.

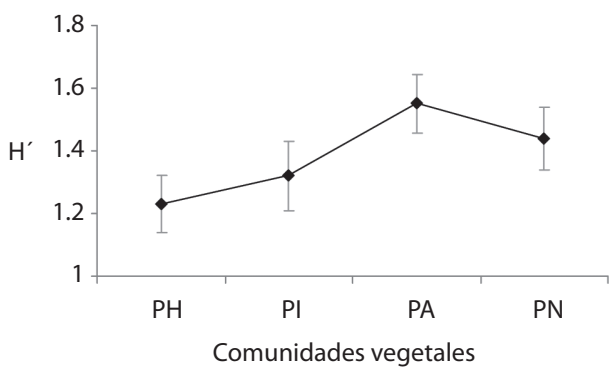

Fig. 3. Índice de Shannon-Wiener $\left(\mathrm{H}^{\prime}\right)$ (promedio $( \pm \mathrm{ES})$ para las comunidades de acridios presentes en las diferentes comunidades vegetales de Laprida, Buenos Aires, durante 2005-2010.

PH: pastizales halófilos, PI: pasturas implantadas, PA: pastizales alterados, $\mathrm{PN}$ : pastizales nativos.

Fig. 3. Shannon-Wiener (H') index (average value $( \pm \mathrm{SE})$ for grasshopper communities in different plant communities in Laprida, Buenos Aires, during 2005-2010.

PH: halophilous grasslands, PI: implanted pastures, PS: disturbed grasslands, PN: native grasslands.

abundancia relativa, mostró valores menores (Cuadro 6). Las comunidades de acridios de los pastizales alterados y las pasturas implantadas fueron las más semejantes. Considerando ambos índices, las comunidades de acridios de los pastizales nativos y de los pastizales halófilos serían las más diferentes (Cuadro 6).

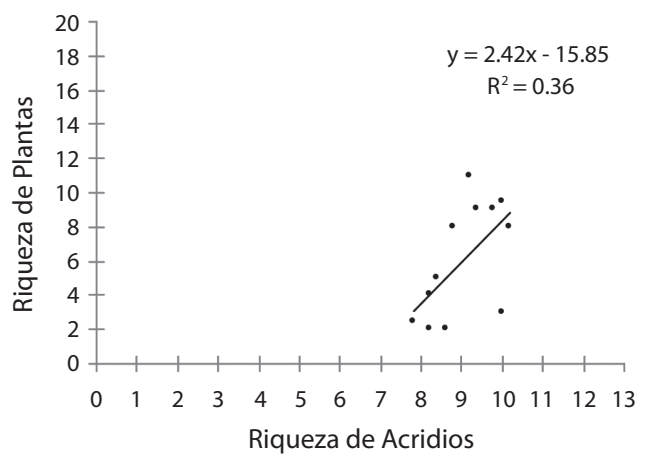

Fig. 4. Regresión entre la riqueza de plantas y la riqueza de acridios en Laprida, Buenos Aires, 2005-2010.

Fig. 4. Regression between plant species richness and grasshopper richness in Laprida, Buenos Aires, 2005-2010.

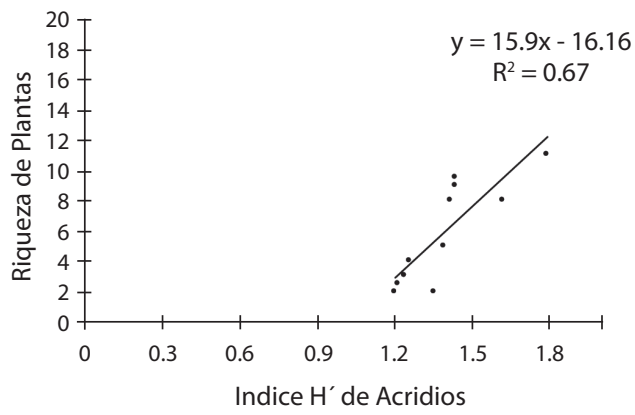

Fig. 5. Regresión entre la riqueza de plantas y el índice $\mathrm{H}^{\prime}$ de Shannon-Wiener de acridios en Laprida, Buenos Aires, 2005-2010.

Fig. 5. Regression between plant species richness and grasshopper diversity index (H') in Laprida, Buenos Aires, 2005-2010.

Con respecto a la distribución de las especies, considerando todos los sitios/años $(\mathrm{n}=321)$, cinco especies presentaron una "amplia" distribución (Covasacris pallidinota, Dichroplus elongatus, Dichroplus maculipennis, Borellia bruneri y Borellia pallida) (Cuadro 4). Las primeras cuatro también fueron las más abundantes, mientras que $B$. pallida presentó una abundancia menor. La mayoría de las especies (12) mostró una distribución restringida, con abundancias menores al $3 \%$, y cinco especies exhibieron una distribución intermedia (26-50\%) (Aleuas lineatus, 


\section{CUADRO 6}

Indices de similitud aplicados para las comunidades de acridios

presentes en diferentes comunidades vegetales de Laprida, Buenos Aires, durante 2005-2010

TABLE 6

Similarity index applied to grasshopper communities present in different plant communities in Laprida, Buenos Aires, during 2005-2010

\begin{tabular}{lcccc}
$2005-2010$ & \multicolumn{3}{c}{ Índices de Similitud } & \\
& Especies comunes & Jaccard & Sørenson Cualitativo & Sørenson Cuantitativo \\
PH vs PN & 14 & 0.67 & 0.80 & 0.47 \\
PH vs PD & 14 & 0.74 & 0.85 & 0.60 \\
PH vs PI & 14 & 0.82 & 0.90 & 0.73 \\
PI vs PN & 16 & 0.80 & 0.89 & 0.69 \\
PI vs PD & 15 & 0.79 & 0.88 & 0.74 \\
PN vs PD & 16 & 0.73 & 0.84 & 0.71 \\
\hline
\end{tabular}

PH: pastizales halófilos, PN: pastizales nativos, PD: pastizales alterados, PI: pasturas implantadas.

Baeacris pseudopuntulactus, Dichroplus conspersus, Dichroplus pratensis, Sinipta dalmani), con abundancias que variaron entre el $2 \%$ y el 8\% (Cuadro 4). El resultado del análisis de regresión efectuado entre el Ln (logaritmo natural) de la abundancia media y la cantidad de sitios ocupados fue altamente significativo $(p<0.0001)$ (Fig. 6). En general, las especies con mayor abundancia tuvieron una

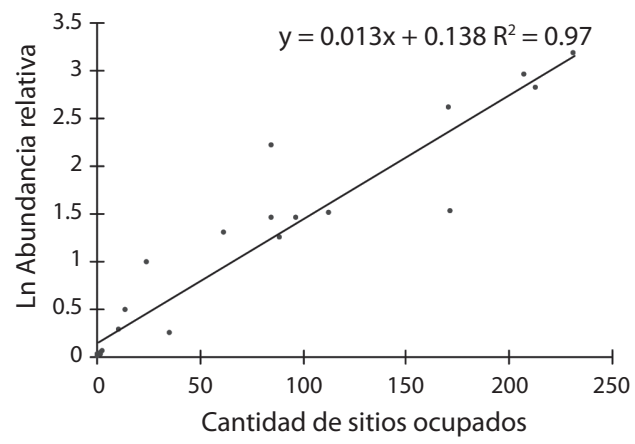

Fig. 6. Regresión entre el logaritmo natural (Ln) de la abundancia relativa y la cantidad de sitios ocupados por especie de acridio en Laprida, Buenos Aires, 2005-2010.

Fig. 6. Regression between natural logarithm (Ln) of relative abundance and the number of sites occupied by grasshopper species in Laprida, Buenos Aires, 2005-2010. mayor frecuencia de aparición, mostrando una mayor distribución.

\section{DISCUSIÓN}

A nivel mundial, se conocen 25000 especies de Orthoptera (Eades et al. 2011) y si bien este orden tiene una distribución cosmopolita, la mayor diversidad se encuentra en áreas tropicales (Barrientos Lozano 2005). En Argentina, se han registrado 203 especies de la superfamilia Acridoidea, siendo la familia Acrididae la de mayor diversidad con 142 especies, seguida de la familia Romaleidae con 36 (Carbonell et al. 2006; Eades et al. 2011). Las especies de acridios registradas en este estudio se encuentran incluidas dentro de estas dos familias, siendo la familia Acrididae la más importante porque representa el $95.45 \%$ del total de especies colectadas. De las seis subfamilias de Acridoidea representadas la más diversa fue Melanoplinae, seguida de Gomphocerinae y de Acridinae. Las especies pertenecientes a estas tres subfamilias se encuentran generalmente asociadas a sistemas de pastizal (Carbonell et al. 2006, Pocco et al. 2010). De acuerdo a las citas registradas para la provincia de Buenos Aires por Carbonell et al. (2006) en este estudio se colectó el $50 \%$ de las especies de las 
subfamilias Gomphocerinae y Acridinae y el $38 \%$ de las especies de melanoplinos. Estudios previos realizados en otras zonas de la región pampeana (Sánchez \& De Wysiecki 1993, Cigliano et al. 2000, De Wysiecki et al. 2000, De Wysiecki et al. 2004) y en otras regiones del país, como pastizales del centro de Santa Fe (Zequín et al. 1999) y del Chaco Húmedo Oriental (Pocco et al. 2010), coinciden en que la familia Acrididae fue la más representativa en sus colectas, siendo también las subfamilias Melanoplinae y Gomphocerinae las más diversas.

La riqueza de especies de acridios registrada en este estudio es coincidente con la reportada en otros estudios realizados en diferentes sectores de la región pampeana (Sánchez \& De Wysiecki 1993, Cigliano et al. 2000, De Wysiecki et al. 2000, De Wysiecki et al. 2004), y con lo expresado por Otte (1976), quien señala que la riqueza promedio de especies en pastizales de la región pampeana tiene un rango aproximado de 6 a10 especies, siendo estos valores menores a los estimados para diversos sistemas de pastizal de EEUU. Kemp (1992b) en estepas del Oeste de EEUU determinó entre 10 y 17 especies y Joern (2005) en pastizales de Manhattan, EEUU, estimó un promedio de $16.2 \pm 7.8$ especies, con un rango de 6 a 24 especies por sitio.

La diversidad de un área está constituida por dos componentes principales, la riqueza de especies y la equitatividad o distribución de la abundancia o biomasa de las diferentes especies de una comunidad (Wilsey \& Potvin 2000). Estos dos componentes pueden o no correlacionarse, indicando que las medidas de equitatividad pueden proporcionar información significativa sobre la variación en la diversidad de un área, independientemente de la información aportada por la variación en la riqueza de especies (Hillebrand et al. 2008). En el presente estudio, entre ambas variables se detectó una correlación positiva significativa, indicando que a medida que aumentó la cantidad de especies colectadas en los diferentes sitios también lo hizo la equitatividad. Stirling \& Wilsey (2001) señalan que la correlación entre estas variables dependerá de los organismos en cuestión, ya que según el análisis de diferentes estudios la correlación entre estas variables suele ser fuertemente positiva en los invertebrados, como se observó en el presente trabajo, débilmente positiva en vertebrados y negativa en las plantas.

Varios estudios han mostrado que la diversidad de insectos herbívoros estaría relacionada con características de la vegetación y/o complejidad estructural de las misma (Stamps \& Linit 1998). En este trabajo se registró una relación significativa entre la riqueza de acridios y los valores del índice de diversidad de Shannon-Wiener con la variable riqueza de plantas, indicando que los valores de estos índices son mayores en los sitios que presentan una mayor riqueza de especies de plantas, como son los pastizales alterados. En otros pastizales de la región pampeana, Torrusio et al. (2002) no registraron una relación significativa entre las variables riqueza de acridios y riqueza de plantas, indicando que probablemente la riqueza de especies de acridios no se encuentre en función de la riqueza de plantas de un determinado lugar. Branson (2011) en pastizales de Dakota, EEUU, registró una asociación positiva entre la riqueza de plantas de los diferentes ambientes y la diversidad de acridios estimada a través del índice de Shannon-Wiener, pero no observó una relación significativa entre las variables riqueza de acridios y riqueza de plantas. En dicho trabajo, el autor indica que los tipos de plantas y las asociaciones entre éstas podrían explicar mejor la cantidad de especies de acridios presentes en una determinada comunidad vegetal que la riqueza de plantas en si misma.

Con respecto a los índices de similitud aplicados, se pudo observar que las diferentes comunidades vegetales comparten un alto porcentaje de especies de acridios. Los índices cualitativos mostraron que las comunidades de acridios presentes en los diferentes pastizales se asemejan en un rango del 67 al 82\%, según Jaccard y entre el 80-90\%, según Sorensen. Los valores del índice cuantitativo de Sorensen fueron menores, entre el 47 y el 74\%, lo cual índica que si bien las especies se pueden presentar 
en los diferentes ambientes lo hacen con abundancias disímiles, esto podría indicar posibles preferencias del hábitat. En Nebraska, EEUU, Joern (1982) registró que el grupo de especies de acridios encontrado en diferentes pastizales fue básicamente el mismo, pero que las especies se presentaban con abundancias diferentes.

Con respecto a la distribución de las especies, al considerar todos los sitios, se observó que la mayoría de las especies (54.5\%) tuvo una distribución restringida, el $22.7 \%$ una distribución intermedia y el mismo porcentaje $(22.7 \%)$ una distribución amplia. En general la abundancia y la distribución de las especies están positivamente correlacionadas: las especies que tienen una distribución más amplia tienden a ser más abundantes que aquellas cuya distribución es más restringida (Brown 1984, Hanski et al. 1993). De las cinco especies con mayor distribución, cuatro fueron las más abundantes, B. bruneri, C. pallidinota, D. elongatus y D. maculipennis. Borellia pallida tuvo una abundancia relativa inferior. El patrón de distribución de $B$. pallida no es congruente con la relación "mayor distribución mayor abundancia" propuesta por Hanski et al. (1993). Cigliano et al. (2000) registraron una situación similar para Baeacris punctulactus. Esta especie tuvo una amplia distribución, pero al igual que $B$. pallida su abundancia relativa fue baja. Los autores consideraron que podría ser una especie "numéricamente rara", calificación que también podría considerarse para $B$. pallida luego de cinco años de muestreos.

Hasta el presente, los tres estudios de distribución de acridios realizados en Argentina siguieron la categorización propuesta por Kemp (1992a). Cigliano et al. (2000) registraron en 12 sitios del Oeste de la región pampeana entre 1994-99, cinco especies con amplia distribución, nueve con distribución intermedia y 20 con una distribución rara. Las cinco especies con mayor distribución fueron $D$. elongatus, $D$. pratensis, Staurorhectus longicornis, A. lineatus y B. punctulatus. De Wysiecki et al. (2000), en cinco sitios del noreste de la provincia de La Pampa, recolectaron un total de 19 especies, nueve de las cuales fueron de distribución rara, siete de distribución intermedia y tres ampliamente distribuidas (D. pratensis, Neopedies brunneri y Euplectrotettix ferrugineus). A diferencia de los resultados obtenidos en Laprida, $D$. elongatus y $B$. bruneri fueron especies de distribución rara. De Wysiecki et al. (2004), en Benito Juárez, encontraron 17 especies con distribución rara, cinco especies de distribución intermedia y una sola especie ampliamente distribuida (D. elongatus).

Podemos concluir que, independientemente de la escala utilizada para estimar la distribución de las especies, las comunidades de acridios de los pastizales de la región pampeana coinciden con el patrón propuesto por Kemp (1992a), en el cual la mayoría de las especies tienen una distribución intermedia o restringida y pocas son las que presentan una distribución amplia. Al respecto, Kemp (1990) indica que los acridios conforman un gran grupo de especies intermedias y que su abundancia y dominancia están influenciadas significativamente por diferencias asociadas al tipo de hábitat.

Para finalizar es importante destacar que de las cinco especies más abundantes, tres: B. bruneri, D. elongatus y D. maculipennis, son consideradas perjudiciales para el agro en Argentina. Dichroplus elongatus y D. maculipennis se encuentran dentro de las especies de acridios económicamente más dañinas, siendo sus explosiones poblacionales fenómenos recurrentes en diferentes regiones del país (Lange et al. 2005, Mariottini et al. 2011b), donde no solo compiten con el ganado por el forraje disponible sino que consumen y destruyen pasturas y diferentes cultivos, produciendo pérdidas económicas de gran magnitud para los productores de la zona (Lange et al. 2005, De Wysiecki et al. 2011).

El presente trabajo profundiza en el conocimiento sobre la diversidad, abundancia y distribución de acridios en una de las áreas histórica y actualmente más afectadas por estos insectos. Creemos que estudios sostenidos en espacio y tiempo son fundamentales a la hora de proveer bases tendientes para realizar un manejo racional de las especies de acridios consideradas plagas. 


\section{AGRADECIMIENTOS}

Esta investigación fue financiada en parte por el "Fondo para la Investigación Científica y Tecnológica (FONCYT, proyecto PICT 2006/679)" y "Comisión de Investigaciones Científicas (CIC) de la provincia de Buenos Aires".

\section{RESUMEN}

Los acridios son componentes nativos de los pastizales, dichos sistemas han sido modificados debido a la intensificación de las actividades agrícola-ganaderas y por lo tanto se considera importante estudiar las comunidades de acridios asociadas. Se estudió la diversidad y distribución de acridios en el Sur de la región pampeana a través de muestreos realizados en las comunidades vegetales más representativas del partido de Laprida, provincia de Buenos Aires, durante cinco temporadas (2005-2010). Se recolectaron 22 especies. La subfamilia Melanoplinae fue la más diversa (ocho especies). La mayor cantidad de especies se observó en los pastizales nativos (18). La diversidad en los pastizales alterados $(1.75 \pm 0.096)$ fue superior $(\mathrm{p}<0.05)$ a la de los pastizales halófilos $(1.34 \pm 0.12)$. La riqueza de plantas en los pastizales nativos y los pastizales alterados fue superior $(\mathrm{p}<0.05)$ a la de las demás comunidades. Se registro una relación positiva entre la riqueza de plantas y las variables riqueza y diversidad de acridios. La similitud entre las comunidades de acridios estimada por los índices cualitativos fue superior al $60 \%$. En general, las especies que tuvieron una mayor frecuencia de aparición, mostraron una mayor abundancia y distribución. Covasacris pallidinota, Dichroplus elongatus, D. maculipennis, Borellia bruneri y $B$. pallida fueron las de mayor distribución, cinco especies tuvieron una distribución intermedia y la mayoría (12) una distribución restringida.

Palabras clave: Acridios, pastizales, riqueza de especies, índices de diversidad, distribución.

\section{REFERENCIAS}

Altieri, M. 1983. Agroecología. Bases Científicas de la Agricultura Alternativa. California, Berkeley, EEUU.

Barrientos Lozano, L. 2005. ¿Qué son las langostas y los saltamontes? Clasificación de los Orthoptera (Caelifera sensu stricto). Especies plaga en México, p. 1-29. In L. Barrientos Lozano \& P. Almaguer (eds.). Manejo integrado de la langosta centroamericana y acridoideos plaga en América Latina. Instituto Tecnológico de Ciudad Victoria, Tamaulipas, México.
Batista, W.B., R.J.C. León \& S.B. Perelman. 1988. Las comunidades vegetales de un pastizal natural de la región de Laprida, Prov. de Buenos Aires, Argentina. Phytoeconología 16: 519-534.

Batista, W.B., M.A. Taboada, R.S. Lavado, S.B. Perelman \& R.J.C. León. 2005. Asociación entre comunidades vegetales y suelos en el pastizal de la Pampa Deprimida, p. 113-129. In M. Oesterheld, M.R. Aguiar, C.M. Ghersa \& J.M. Paruelo (eds.). La heterogeneidad de la vegetación de los agroecosistemas. Un homenaje a Rolando León. Editorial Facultad de Agronomía, Buenos Aires, Argentina.

Belovsky, G.E. 2000. Do grasshoppers diminish grassland productivity? A new perspective for control based on conservation, p. 7-29. In J.A. Lockwood, A.V. Latchininsky \& G. Sergeev (eds.). Grasshoppers and Grassland Health: Managing Grasshopper Outbreaks without Risking Environmental Disaster. Kluwer Academic, Boston, EEUU.

Branson, D.H., A. Joern \& G.A. Sword. 2006. Sustainable Management of Insect Herbivores in Grassland Ecosystems: New Perspectives in Grasshopper Control. Bioscience 56: 743-755.

Branson, D.H. 2011. Relationships between plant diversity and grasshopper diversity and abundance in the Little Missouri National Grassland. Psyche (Disponible en línea: http://www.hindawi.com/journals/ psyche/2011/748635/).

Brown, J.H. 1984. On the relationship between abundance and distribution of species. Am. Nat. 124: 255-79.

Carbonell, C.S., M.M. Cigliano \& C.E. Lange. 2006. Acridomorph (Orthoptera) species of Argentina and Uruguay. CD-ROM. Publications on Orthopteran Diversity, The Orthopterists Society at the "Museo de La Plata", Argentina.

Chaneton, E.J. 2005. Factores que determinan la heterogeneidad de la comunidad vegetal en diferentes escalas espaciales, p. 19-42. In M. Oesterheld, M.R. Aguiar, C.M. Ghersa \& J.M. Paruelo (eds.). La heterogeneidad de la vegetación de los agroecosistemas. Un homenaje a Rolando León. Editorial Facultad de Agronomía, Buenos Aires, Argentina.

Cigliano, M.M., M.L. De Wysiecki \& C.E. Lange. 2000. Grasshopper (Orthoptera, Acrididae) species diversity in the pampas, Argentina. Divers. Distrib. 6: 81-91.

Daubenmire, R. 1959. A canopy cover method of vegetational analysis. Northwest Sci. 33: 43-66.

De Wysiecki, M.L., N. Sánchez \& S. Ricci. 2000. Grassland and shrubland grasshopper community composition in northern La Pampa province, Argentina. J. Orthop. Res. 9: 211-221.

De Wysiecki, M.L., S. Torrusio \& M.M. Cigliano. 2004. Caracterización de las comunidades de acridios 
del partido de Benito Juárez, sudeste de la provincia de Bs. As, Argentina. Rev. Soc. Entomol. Argent. 63: 87-96.

De Wysiecki, M.L., M. Arturi, S. Torrusio \& M.M. Cigliano. 2011. Influence of weather variables and plant communities on grasshopper density in the Southern Pampas, Argentina. J. Insect Sci. 11: 109 (Disponible en línea: www.insectscience.org/11.109).

Eades, D.C., D. Otte, M.M. Cigliano \& H. Braun. Orthoptera Species File Online. Version 2.0/4.0. (Disponible en línea: www.Orthoptera.SpeciesFile.org).

Facelli, J.M. 1988. Response to grazing after nine years of cattle exclusion in a flooding pampa grassland, Argentina. Vegetatio 78: 21-25.

Fielding, D.J. \& M.A. Brusven. 1993. Grasshoppers (Orthoptera: Acrididae) community composition an ecological disturbance on Southern Idaho rangeland. Environ. Entomol. 22: 71-81.

Fielding, D.J. \& M.A. Brusven. 1995. Ecological correlates between rangeland grasshopper (Orthoptera: Acrididae) and plant communities of southern Idaho. Environ. Entomol. 24: 1432-1441.

Ghersa, C.M. 2005. La Sucesión ecológica en los Agrosistemas pampeanos. Sus modelos y Significado agronómico, p. 195-212. In M. Oesterheld, M.R. Aguiar, C.M. Ghersa \& J.M. Paruelo (eds.). La heterogeneidad de la vegetación de los agroecosistemas. Un homenaje a Rolando León. Editorial Facultad de Agronomía, Buenos Aires, Argentina.

Hanski, I., J. Kouki \& A. Halkka. 1993. Three explanations of the positive relationship between distribution and abundance of species, p. 108-116. In R.E. Ricklefs $\&$ D. Schluter (eds.). Species diversity in ecological communities: historical and geographical perspectives. University of Chicago, Chicago, EEUU.

Hill, M.O. \& H.G. Gauche. 1980. Detrended correspondence analysis: an improved ordination technique. Vegetatio 42: 47-58.

Hillebrand, H., D.M. Bennett \& M.W. Cadotte. 2008. Consequences of dominance: a review of evenness effects on local and regional ecosystem processes. Ecology 89: 1510-1520.

Insausti, P., E.J. Chaneton \& A.A. Grimoldi. 2005. Las inundaciones modifican la estructura y dinámica de la vegetación en los pastizales de la Pampa Deprimida, p. 253-269. In M. Oesterheld, M.R. Aguiar, C.M. Ghersa \& J.M. Paruelo (eds.). La heterogeneidad de la vegetación de los agroecosistemas. Un homenaje a Rolando León. Editorial Facultad de Agronomía, Buenos Aires, Argentina.

Joern, A. 1982. Distributions, Densities and Relative Abundances of Grasshoppers (Orthoptera: Acrididae) in a Nebraska Sandhills Prairie. Prairie Naturalist 14: 37-45.
Joern, A. \& S.B. Gaines. 1990. Population dynamics and regulation in grasshoppers, p. 415-483. In R.F. Chapman \& A. Joern (eds.). Biology of Grasshoppers. John Wiley \& Sons, Nueva York, EEUU.

Joern, A. 2005. Disturbance by Fire and Bison Grazing Modulate Grasshopper Assemblages in Tallgrass Prairie. Ecology 86: 861-873.

Jonas, J.L. \& A. Joern. 2007. Grasshopper (Orthoptera: Acrididae) communities respond to fire, bison grazing and weather in North American tallgrass prairie: a longterm study. Oecologia 153: 699-711.

Kemp, W.P. 1990. Habitat and insect biology revisited. Am. Entomol. 36: 44-49.

Kemp, W.P. 1992a. Temporal variation in rangeland grasshopper (Orthoptera: Acrididae) communities in the steppe region of Montana, USA. Can. Entomol. 124: 437-450.

Kemp, W.P. 1992b. Rangeland grasshopper (Orthoptera: Acrididae) community structure: a working hypothesis. Environ. Entomol. 21: 461-470.

Kemp, W.P. \& M.M. Cigliano. 1994. Drought and rangeland grasshopper species diversity. Can. Entomol. 126: 1075-1092.

Lange, C.E., M.M. Cigliano, M.L. De Wysiecki. 2005. Los acridoideos de importancia económica en la Argentina, p. 93-135. In L. Barrientos Lozano \& P. Almaguer (eds.). Manejo integrado de la langosta centroamericana y acridoideos plaga en América Latina. Instituto Tecnológico de Ciudad Victoria, Tamaulipas, México.

Larson, D.P., K.M. O'neill \& W.P. Kemp. 1999. Evaluation of the accuracy of sweep sampling in determining grasshopper (Orthoptera: Acridoidea) community composition. J. Agron. Urban Entomol. 16: 207-214.

Mariottini, Y., M.L. De Wysiecki \& C.E. Lange. 2011a. Seasonal occurrence of life stages of Grasshopper (Orthoptera: Acridoidea) in the Southern Pampas, Argentina. Zool. Studies 50: 737-744.

Mariottini, Y., M.L. De Wysiecki \& C.E. Lange. 2011 b. Postembryonic development and consumption of the melanoplines Dichroplus elongatus Giglio-Tos and Dichroplus maculipennis (Blanchard) (Orhtoptera: Acrididae: Melanoplinae) under laboratory conditions. Neot. Entomology 40: 190-196.

Milchunas, D.G. \& W.L. Laurenroth. 1993. Quantitative effects of grazing on vegetation and soils over a global range of environments. Ecol. Monog. 63: 327-366.

Nai-Bregaglio, M., E. Pucheta \& M. Cabido. 2002. El efecto del pastoreo sobre la diversidad florística y estructural en Pastizales de montaña del centro de Argentina. Rev. Chil. Hist. Nat. 75: 613-623.

Omancini, M., P. Tognetti, H. Trebino \& E.J. Chaneton. 2005. La sucesión postagrícola en la Pampa Interior: invasión y dominancia de plantas exóticas durante 
los primeros 20 años, p. 215-231. In M. Oesterheld, M. Aguiar, C.M. Ghersa \& J. Paruelo (eds.). La heterogeneidad de la vegetación de los agroecosistemas. Un homenaje a Rolando León. Editorial Facultad de Agronomía, Buenos Aires, Argentina.

Otte, D. 1976. Species richness patterns of New World desert grasshoppers in relation to plant diversity. J. Biogeog. 3: 197-209.

Perelman, S.B., R.J.C. León \& M. Oesterheld. 2001. Cross-scale vegetation patterns of Flooding Pampa Grasslands. J. Ecol. 89: 562-577.

Pfadt, R.E. 1982. Density and diversity of grasshoppers (Orthoptera: Acrididae) in an outbreak on Arizona rangeland. Environ. Entomol. 11: 690-694.

Pocco, M.E., M.P. Damborsky \& M.M. Cigliano. 2010 Comunidades de ortópteros (Insecta, Orthoptera) en Pastizales del Chaco Oriental Húmedo, Argentina. Anim. Biodivers. Conserv. 33: 119-129.

Ronderos, R.A. 1985. Consideraciones sobre la biogeografía de los Melanoplinae en Sudamerica (Orthptera, Acrididae). Proc. 3rd. Trienial Meeting, Pan American Acrid. Society 53-54.

Root, R.B. 1973. Organization of a plant-arthropod association in simple and diverse habitats: the fauna of collards (Brassicae oleraceae). Ecol. Monogr. 43: 95-124.

Sala, O.E., V.A. Deregibus, T. Schlichter \& H. Alippe. 1981. Productivity dynamics of a native temperate grassland in Argentina. J. Range Manage. 34: 48-51.

Sala, O.E., M. Oesterheld, R.J.C. León \& A. Soriano. 1986. Grazing effect upon plant community structure in subhumid grasslands of Argentina. Vegetatio 67: 27-32.
Sánchez, N.E. \& M.L. De Wysiecki. 1993. Abundancia y diversidad de acridios (Orthoptera: Acrididae) en pasturas de la Provincia de La Pampa, Argentina. Rev. Invest. Agropecu. INTA 24: 29-39.

Soriano, A, R.J.C. León, O.E. Sala, R.S. Lavado, V.A. Deregibus, M.A. Cahuepé, O.A. Scaglis, C.A. Velázquez \& J.H. Lemcoff. 1992. Río de la Plata grasslands, p. 367-407 In R.T. Coupland (ed.). Ecosystems of the world. Natural grasslands. Elsevier, Nueva York, EEUU.

Stamps, W.T. \& M.J. Linit. 1998. Plant diversity and arthropod communities: Implications for temperate agroforestry. Agrofor. Syst. 39:73-89.

Stirling, G. \& B. Wilsey. 2001. Empirical relationships between species richness, evenness and proportional diversity. Am. Nat. 158: 286-299.

Torrusio, S.A, M.M. Cigliano \& M.L. De Wysiecki. 2002. Grasshopper (Orthoptera: Acrididae) and plant community relationships in the Argentine pampas. J. Biogeog. 29: 221-229.

Wilsey, B.J. \& C. Potvin. 2000. Biodiversity and ecosystem functioning: importance of species evenness in an old field. Ecology 81: 887-892.

XLSTAT-PRO 7.5.3. 2005. (Disponible en línea: http:// www.xlstat.com/en/home). Addinsoft Inst Inc, Nueva York, EEUU.

Zequin, L., R. Beltrame, S. Luiselli, C. Salto \& R. Strasser. 1999. Abundancia y diversidad de tucuras (Orthoptera: Acridoidea) en el centro oeste de Santa Fe y centro este de Córdoba. INTA EEA Rafaela. Anuario 1999.

Zhong-Wei, G., L.I. Hong-Chang \& Ya-Ling Gan. 2006. Grasshoppers (Orthoptera: Acrididae) biodiversity and grassland ecosystems. Insect Sci.13: 221-227. 